\title{
The Role of Airway Epithelial Cells in Response to Mycobacteria Infection
}

\author{
Yong Li, 1, 2 Yujiong Wang, ${ }^{1,2}$ and Xiaoming Liu', ${ }^{1,2}$ \\ ${ }^{1}$ Key Laboratory of the Ministry of Education for the Conservation and Utilization of Special Biological Resources of Western China, \\ Ningxia University, Yinchuan, Ningxia 750021, China \\ ${ }^{2}$ College of Life Science, Ningxia University, Yinchuan, Ningxia 750021, China
}

Correspondence should be addressed to Yujiong Wang, wyj@nxu.edu.cn and Xiaoming Liu, erc1080@gmail.com

Received 31 December 2011; Accepted 15 February 2012

Academic Editor: S. Sozzani

Copyright (C) 2012 Yong Li et al. This is an open access article distributed under the Creative Commons Attribution License, which permits unrestricted use, distribution, and reproduction in any medium, provided the original work is properly cited.

\begin{abstract}
Airway epithelial cells (AECs) are part of the frontline defense against infection of pathogens by providing both a physical barrier and immunological function. The role of AECs in the innate and adaptive immune responses, through the production of antimicrobial molecules and proinflammatory factors against a variety of pathogens, has been well established. Tuberculosis (TB), a contagious disease primarily affecting the lungs, is caused by the infection of various strains of mycobacteria. In response to mycobacteria infection, epithelial expression of Toll-like receptors and surfactant proteins plays the most prominent roles in the recognition and binding of the pathogen, as well as the initiation of the immune response. Moreover, the antimicrobial substances, proinflammatory factors secreted by AECs, composed a major part of the innate immune response and mediation of adaptive immunity against the pathogen. Thus, a better understanding of the role and mechanism of AECs in response to mycobacteria will provide insight into the relationship of epithelial cells and lung immunocytes against TB, which may facilitate our understanding of the pathogenesis and immunological mechanism of pulmonary tuberculosis disease.
\end{abstract}

\section{Introduction}

The lung is an organ lined by numerous distinct types of epithelial cells in different anatomical regions. Pulmonary epithelium was initially thought of as a complicated physical barrier to block potentially harmful inhaled bacilli and substances from tissue invasion. However, increasing evidence has demonstrated that epithelial cells also play critical functions in initiating and expanding airway host defense mechanisms in the lung and providing the initial defense against inhaled microorganisms. Furthermore, these epithelial cells are capable of not only regulating innate immunity but also producing functional molecules that physically interacted with immunocytes to activate adaptive immunity $[1,2]$. These epithelial cell-derived antimicrobial molecules and/or peptides were multifunctional agents capable of linking the innate and adaptive immune responses [3]. In response to invasion of pathogens, airway epithelial cells (AECs) secrete various microorganism killing effectors, such as mucins antimicrobial peptides (AMPs) and reactive oxygen species (ROS), into the airway lumen to control the composition of airway surface liquid (ASL). These cells also produce proinflammatory cytokines, growth factors, and chemokines that recruit and activate phagocytes to the site of infection eliminating pathogens by phagocytosis. These effectors play key roles in innate immunity of the airway against microorganism invasion and pathogenesis of pulmonary inflammatory diseases caused by chronic bacterial infection [4].

Mycobacterium tuberculosis (Mtb) is an extremely dangerous pathogen that primarily infects the lung and is known for its capability to escape innate immune effector cells (such as macrophages and airway epithelial cells) causing tuberculosis in humans and animals [5]. The AECs are one of the first host cells encountering invaded mycobacterial pathogens, despite increasing evidence, which demonstrates the potential roles of these cells in the tuberculosis (TB) pathogenesis, as well as the innate and adaptive immune responses against the infection [6-9]. The exact mechanism of epithelial mediated host defense and the clinical relevance 
of the epithelial cell-mediated immune responses remain poorly understood. This paper focuses on current knowledge regarding the roles of AECs in response to mycobacteria infection. An emphasis was placed on the recently recognized functions of epithelial cells in innate and adaptive immunity against mycobacteria, particularly the pathogen recognition and host defense of AECs to Mtb.

\section{Airway Epithelial Cells and Pathogen Recognition of Mycobacteria}

Mycobacterium utilizes multiple strategies for establishing infection of the lung, including adhering, invasion, and replication in alveolar macrophages $[6,10,11]$. An important step of infection is the initial contact of a pathogen to host cells including epithelial cells. The innate immune response is the first line of host defense responsible for immediate recognition and control of pathogen invasion. The lung microenvironment contains an intricate milieu of pattern recognition molecules in the innate immune system that contribute to the host primary response to inhaled pathogens such as Mtb. It has been suggested that Mtb affects the result of the bacillus-host interaction with various recognition molecules $[12,13]$. The family of Tolllike receptors (TLRs) and surfactant proteins (SPs) of AECs play a key role in the recognition and binding of the pathogen to epithelial cells during Mtb infection. Additionally, other pattern recognition receptors (PRRs) such as NOD2, Dectin1, c-type lectin receptors (CLPs), mannose receptor, and DCSIGN are also believed to be involved in the recognition of Mtb [14].

\subsection{Role of Epithelial TLRs in the Recognition of Mycobac-} teria. Genetic association studies revealed that TLR variants contributed to the susceptibility of humans to TB [15]. The genes of various TLRs have been demonstrated to be predominantly expressed in immunocyte such as macrophages, dendritic cells (DCs), B lymphocytes, monocytes, and natural killer (NK) cells. The AECs have also been shown to constitutively express TLRs which are one of the most important PRRs for pathogen recognition. These TLRs mediate the host-pathogen interaction through pathogenassociated molecular patterns (PAMPs) and initiate appropriate signaling before immune cells are recruited to the airways. To date, thirteen distinct mammalian TLRs have been discovered, ten of which recognize PAMPs, and have been identified in humans (designated TLR-1 to 10). The expression of TLR-1, TLR-2, TLR-4, TLR-5, TLR-6, and TLR10 was located at the apical cell membrane of AECs where they mainly recognize proteins, lipoproteins, and the polysaccharides of the bacteria. In addition, the expression of TLR-3, TLR-7, TLR-8, and TLR-9 was detected in cytoplasmic compartments where they scavenge different types of nucleic acids from viruses [16-18]. Each TLR recognizes a unique molecular pattern and a variety of ligands to mediate the production of the appropriate cytokines to produce an effective immune response.
In response to the infection of $\mathrm{Mtb}$, airway epithelial activation of NF- $\kappa \mathrm{B}$ and other associated signaling molecules are activated via TLR-mediated signaling pathways. Previous studies have demonstrated functional TLR-1-6 and TLR-9 expressed in human bronchial epithelial cells [13] and several TLRs involved in the immune response of AECs against Mtb infection (Table 1). Furthermore, human bronchial epithelium was capable of regulating its sensitivity to recognize microbes by management of the TLR's expression levels to control microbial recognition in mucosal compartments [13]. Mice deficient of myeloid differentiation factor 88 (MyD88), a TLR mediator molecule, were approximately twofold more susceptible to lethal doses of Mtb infection relative to wild-type mice even though MyD88-deficient mice vaccinated with Mycobacterium bovis Bacille CalmetteGuérin (BCG) were able to confer substantial protection from acute Mtb infection [19]. This study suggests that MyD88-dependent TLR signaling might be dispensable to adaptive immune responses to $\mathrm{Mtb}$, but crucial for the innate immune response to control Mtb infection. Among these, TLRs, TLR-2, TLR-4, TLR-6, and MyD88 played the most prominent roles in the initiation of the immune response against Mtb [20].

TLR-2 is expressed throughout the human airway epithelium, predominantly in the noncolumnar epithelial and alveolar cells [21], which mediated responses to a variety of bacterial components including the lipoproteins of $\mathrm{Mtb}$, Gram-positive and Gram-negative bacteria [22]. In response to Mtb infection, TLR-2 was specifically targeted to a $19 \mathrm{kDa}$ lipoprotein and lipoarabinomannan of this pathogen, and its activity, ligand specificity, and capacity of signal transduction were mainly determined by a heterodimer formed with TLR2 and other TLR family members, such as TLR- 1 and TLR6 [23]. It has been suggested that the formation of a TLR2 heterodimer with TLR-1 or TLR- 6 has evolved to expand the ligand spectrum to promote the innate immune system for recognition of numerous structures of lipoproteins and lipopeptides presented by different pathogens [24-26]. TLR2-deficient mice infected with live mycobacterial aerosol displayed reduced bacterial clearance, a defective granulomatous response, and development of chronic pneumonia. The pulmonary immune responses in TLR-2-deficient mice showed increased levels of IFN- $\gamma$, TNF- $\alpha$, and IL-12p 40 , as well as the $\mathrm{CD}_{4}^{+}$and $\mathrm{CD} 8^{+}$cell fractions. However, these responses were not sufficient enough to protect the deficient mice from Mtb infection within 5 months after infection suggesting that TLR-2 might be a regulator of inflammation, and the exaggerated immune inflammatory response in these mice resulting from the absence of TLR-2 [27]. In AECs, TLR-2-dependent activation has been demonstrated to function as an indirect role in host defense by inducing the proinflammatory cytokine interleukin (IL) 8 (IL-8) and the antimicrobial peptide human $\beta$-defensin-2 (HBD-2). The action of HBD-2 was believed to act through the chemokine receptor $\mathrm{C}-\mathrm{C}$ chemokine receptor 6 (CCR6) to recruit immature dendritic cells (DCs) and memory $\mathrm{T}$ cells to the site of infection [28]. The neutrophil chemoattractant, IL-8, recruits these cells to the site of pathogen exposure and eventually eliminates the infection [29]. These processes may 
TABLE 1: Evidenced TLR-related AEC in response to Mtb infection.

\begin{tabular}{llcc}
\hline TLRs & Typical Ligands & Cellular location & References \\
\hline TLR1 & Microbial lipoproteins & Cell membrane & {$[93,94]$} \\
TLR2 & Microbial lipoproteins; PGN; LTA; zymosan; & Cell membrane & {$[21,22,24,25,27,94,95]$} \\
TLR3 & dsRNA & Intracellular components & {$[95-97]$} \\
TLR4 & LPS; RSV S protein; retroviral envelope protein & Cell membrane & {$[34]$} \\
TLR5 & Flagellin & Cell membrane & {$[27,33,35,98,99]$} \\
TLR6 & LTA; zymosan; diacyl lipopeptides & Cell membrane & {$[27,93,100]$} \\
TLR7 & ssRNA imidazoquinoline & Intracellular components & {$[95,101]$} \\
TLR8 & ssRNA imidazoquinoline & Intracellular components & {$[101-103]$} \\
TLR9 & Demethylated CpG DNA & Intracellular components & {$[60,98,101,104]$} \\
\hline
\end{tabular}

LPS: lipopolysaccharide; ssRNA: single-stranded RNA; dsRNA: double-stranded RNA; LTA: lipoteichoic acid; PGN: peptidoglycan; RSV: respiratory syncytial virus.

contribute to the resulting adaptive immune response at the site of infection [30].

The function of TLR-4 in both the host's innate and adaptive immunities has been extensively studied, as well as TLR-4 expressed in alveolar and bronchial epithelial cells, and increased in the cells activated by TLR- 4 ligands $[18,31,32]$. However, it is difficult to distinctly define the exact role of TLR-4 against Mtb infection. TLR-4 mutant $(\mathrm{C} 3 \mathrm{H} / \mathrm{HeJ})$ mice intranasally infected with live $\mathrm{Mtb}$ were more susceptible to pathogen and development of pulmonary tuberculosis relative to wild-type $(\mathrm{C} 3 \mathrm{H} / \mathrm{HeN})$ mice, suggesting that TLR-4 may play a protective role in host defense against lung infection by Mtb [33]. This result was consistent with the finding that TLR4 is critical in controlling chronic Mtb infection in mice [34]. However, a controversial report found that TLR-4-mutant mice exhibited increased resistance to low doses of $\mathrm{Mtb}$ infection relative to their wild-type litter mates in a dosedependent manner [35]. Furthermore, TLR-2/TLR-4/TLR-6deficient mice showed their capacity to control chronic $M$. bovis infection, indicating that TLR-2, TLR-4, and/or TLR6 , may be redundant in controlling mycobacteria infection [27]. Synergistic interactions of TLR-2, TLR-6 and TLR-9 in AECs induced high levels of antimicrobial activity against Pseudomonas aeruginosa infection in mice [36], suggesting that multiple TLRs in AECs may produce innate immune responses by the activation of EGFR via a cell signal cascade [37]. These results demonstrate the complicated nature of TLR signaling and hence warrant further studies to establish its protective role against Mtb infection.

2.2. Other Pattern Recognition Receptors. Additional epithelial PRRs have been investigated including Dectin-1, CLPs, NOD2, DC-SIGN, and the mannose receptor. Dectin-1, expressed on the surface of myeloid lineage cells, was initially identified as fungal PRR that bound to $\beta$-glucans and triggered cytokine production by facilitating interaction with TLR-2 [38]. Recent studies that investigated the innate response to mycobacteria demonstrated Dectin-1 expression in alveolar type 2 (ATII) cells that played a critical role in response to Mtb in these non-phagocytic cells [38-40]. A549 cells, an ATII cell line, infected with
Mtb resulted in active induction of Dectin-1 in a TLR-2dependent manner. Furthermore, the induction of Dectin-1 and Mtb-mediated production of ROS was mutually dependent. Additionally, Mtb-dependent Dectin-1 expression was also dependent upon Src kinases. Selective inhibition of Src signaling dramatically decreased Dectin-1 expression. Moreover, Mtb internalization could be partially blocked by silencing Dectin-1 expression, inhibiting Src kinases, or pretreating Mtb with antioxidants [39]. Dectin-1 also has been demonstrated to play a role in promoting Mtb-induced IL$12 \mathrm{p} 40$ production, in which it acts to increase bacterial-host cell interaction and thus enhance the subsequent cytokine response in macrophages and DCs [38]. Further studies revealed that Dectin-1 was required for proinflammatory cytokine release and antimicrobial effects on intracellular mycobacterial growth in A549 cells [41].

\subsection{Surfactant Protein and Mycobacteria Binding. Pul-} monary SP is a multimolecular complex comprised of phospholipids and proteins, which are primarily secreted by airway submucosal cells, Clara cells, and ATII cells into to the extracellular and intra-alveolar space. There are four main surfactant proteins that have overlapping functions, known as SP-A, B, C, and D. Additionally, ATII cells enable synthesis of all four SPs and surfactant lipids that are packaged together in a unique secretory organelle known as the lamellar body [42]. SP physiologically acts to reduce the surface tension of the alveoli by allowing expansion of the lung during inspiration, as well as maintaining alveolar stability by reducing surface tension along the epithelial lining [14, 43]. Human SPs exhibit the capacity to bind Mtb and alter human macrophage-mediated functions in vitro. Although, recent in vivo studies demonstrated no gross defect in SP-A, SP-D, or SP-A/D deficient mice in the uptake or immune control of Mtb. This suggests that SP-A and SP-D were dispensable for immune control of low doses of Mtb challenge in vivo [44]; a number of in vitro and in vivo studies clearly showed that they were involved in host defense functions against Mtb infection [11, 14, 44, 45].

SP-A and SP-D, members of the collectin family, are capable of interacting with pathogens primarily by mediating surfactant function $[46,47]$. Previous studies have revealed 
the ability of SPs to regulate the initial interaction between $\mathrm{Mtb}$ and its intracellular niche, the alveolar macrophage [48]. SP-A has been suggested to play a role in surfactant homeostasis and the host's defense in the lung by its involvement in the early capture and phagocytosis of the pathogenic Mbt by alveolar macrophages [49]. Furthermore, SP-A has been shown in a rat model infected with $M$. bovis BCG to enhance mycobacteria killing by macrophages through a nitric-oxide(NO-) dependent pathway. In this study, SP-A specifically bound to and enhanced the uptake of BCG organisms by macrophages. The ingestion of SP-A-BCG complexes by rat macrophages led to production of inflammatory mediators and increased mycobacteria killing [50]. SP-A preferred to bind the ligands of mannosylated lipoarabinomannan (ManLAM) and lipomannan on the surface of BCG and M. smegmatis, without discrimination between virulent and nonpathogenic strains [49]. The alanine- and proline-rich antigenic (Apa) glycoprotein, expressed with restriction in the Mtb complex strains, was another new potential target for human pulmonary SP-A. This glycoprotein is associated with the Mtb cell wall for a significant amount of time enhancing the attachment of SP-A and possibly accounting for the selective recognition of these strains by SP-A and immune system c-type lectins [51].

SP-D is a lectin that recognizes carbohydrates via its c-type carbohydrate recognition domains (CRDs), and it has been shown to primarily bind the terminal mannosyl oligosaccharides of Mtb to agglutinate bacilli by bridging the carbohydrate binding domains [11]. The conformation of SP-D is extremely important for binding to bacilli. The 321-glutamic acid (Glu) position of human SP-D is a critical site in the binding and regulation of Mtbmacrophage interactions mediated by recognition of $\mathrm{Mtb}$ mannosylated cell wall components [52]. The binding of SP-D and lipoarabinomannan on the surface of Mtb may result in bacterial agglutination, reduced uptake, and bacilli growth impairment within human macrophages. The SP$\mathrm{D}$-mediated inhibition of intracellular growth of $\mathrm{Mtb}$ in macrophages is independent of aggregation and may be a result of increased phagosome-lysosome fusion rather than the generation of a respiratory burst $[53,54]$.

\section{Airway Epithelial Cells and Their Host Defenses}

AECs serve as the first line of defense against pathogen invasion by their function of structural defense as a physical barrier and initiation and augmentation of airway host defense mechanisms. AECs secrete numerous antimicrobial substances, enzymes, ROS, NO, and proinflammatory chemokines and cytokines in response to invasion of a bacterial pathogen such as Mtb, which constitutes a major part of host defense against pathogen infections in the lung.

3.1. Antimicrobial Substances (Peptides). AECs are able to constitutively secret and/or induce secretion of antimicrobial substances including lysozyme, lactoferrin, defensins, collectins, pentraxins, secretory leukocyte protease inhibitor
(SPLI), hepcidin, and cathelicidin (LL-37). These antimicrobial peptides are essential elements of innate immunity. Among them, LL-37, $\beta$-defensin 2, and hepcidin have been demonstrated to play critical roles in innate immunity against mycobacteria infections.

LL-37 is the only member of the cathelicidin family identified to be expressed in human AECs and alveolar macrophages, and it is a major antimicrobial peptide in the innate immune system against Mtb [55]. The expression of LL-37 has been seen in the A549 cells infected or stimulated with mycobacteria such as BCG. BCG-mediated upregulation was influenced by NADPH/ROS, MEK1/2, and p38 MAPK signaling pathways, which played a central role in the regulation of LL-37 gene expression $[56,57]$. Furthermore, the induction of LL-37 expression was also observed with an increasing expression of TLR-2, TLR-4, and TLR-9 signaling in this epithelial cell type [56].

Human $\beta$-defensin-2 (HBD-2) is another known inducible antimicrobial peptide associated with the pathogenesis of human $\mathrm{TB}$ that has the capacity to control the growth and chemotactic activity of $\mathrm{Mtb}$ [58]. Following BCG infection, the expression of HBD2 in human epithelial cells was increased by means of $\mathrm{NF}-\kappa \mathrm{B}$ modulation of increased TNF- $\alpha$ production. In addition to its direct mycobicidal activity, HBD-2 also possesses immunomodulatory functions of stimulating IL-8 production by AECs, as well as enhancing proliferation and cytokine production of $\mathrm{CD} 4^{+} \mathrm{T}$ cell. These studies provide insight into how epithelial-expressed HBD2 enhances the capacity of host defense to control Mtb infection in vivo [59].

Hepcidin (gene name hepcidin antimicrobial peptide $($ HAMP $)$ ), a key negative regulator of iron metabolism in the body, was originally identified as having antimicrobial properties against bacterial infections. The liver is the primary hepcidin-producing organ, although other tissues such as the lung also synthesize this antimicrobe $[60,61]$. Mouse dust cells infected with Mtb exhibit inducible expression of hepcidin mRNA. Similarly, elevated expression of hepcidin may be induced in human alveolar epithelial A549 cells when stimulated by lipoglycans, particularly Mtb mannosecapped lipoarabinomannan, and phosphatidyl-myo-inositol mannosides. The lysed Mtb subcellular fractions and culture filtrate proteins, as well as live BCG, have been shown to stimulate hepcidin mRNA expression in DCs [60]. These results imply that epithelial hepcidin secretion acts as a host defense mechanism against mycobacteria infection.

\subsection{Airway Epithelial Cell Produced Proinflammatory Factors.} Functional PRRs expressed in epithelial cells at different airway mucosal sites provide the capacity of AECs to sense the presence of pathogen infection allowing epithelial cell secretion of various proinflammatory chemokines and cytokines against the pathogen. Mycobacteria activate several signaling events upon contact with AECs that stimulate production of tumor necrosis factor- $\alpha$ (TNF- $\alpha$ ), interleukins, and granulocyte-macrophage colony-stimulating factor (GM-CSF). These proinflammatory factors function 
to recruit and activate phagocytic cells to eradicate organisms and infected cells [62-64]. Several proinflammatory factors produced by AECs in response to mycobacteria infection are listed in Table 2.

TNF- $\alpha$, a cytokine involved in systemic inflammation primarily secreted by the activated macrophages and lung AECs, stimulates the acute phase reaction. Mtb was able to penetrate alveolar epithelium by compromising the epithelial barrier properties and eliciting the production of TNF$\alpha$ after infection, which in turn reduced the bioelectric properties of alveolar epithelium. This process may result in establishing Mtb infection and pulmonary TB [9]. In vitro studies on A549 cells demonstrated that Mtb-induced ROS production may be essential for increased expression of TNF$\alpha$, IL-6, and IL-8 in AECs. This was believed to be mediated by the activation of mitogen-activated protein kinases (MAPKs)/extracellular signal-regulated kinase (ERK) 1/2 and p38 MAPK signaling pathways [65]. Production of TNF$\alpha$, elevating expression of IFN- $\gamma$ and its receptor, as well as the mediator of IFN- $\gamma$ signaling pathway, signal transducer and activator of transcription 1 (STAT1) was seen in Mtbinfected A549 cells [66].

AECs have not previously been considered as an important source of chemokines in pulmonary TB. However, a growing body of evidence has shown that the AECs, particularly the alveolar epithelial cells, are a major source of several chemokine secretions involved in the host response to mycobacteria infection. Furthermore, alveolar epithelial cells may also contribute to the local inflammatory response in human TB by producing chemokines that attract monocytes, lymphocytes, and polymorphonuclear (PMN) cells to the site of infection [67, 68]. IL-8 (CXCL8) is an important activator of the host immune response to Mtb by recruiting inflammatory cells to the site of infection. A signaling pathway comprised of Duox1-TACE-TGF- $\alpha$-EGFR on the surface of AECs was suggested as a defense against BCG infection by production of IL-8 [69]. Intracellular growth was also necessary for mycobacteria to stimulate monocyte chemotactic protein-1 (MCP-1, also known as C-C motif ligand 2 (CCL2)) and IL-8 secretion by alveolar epithelial cells. However, the mycobacterial virulence and rate of intracellular growth did not correlate with chemokine production [67]. Multiple TLRs have been shown to play major roles in IL-8 secretion by activation of EGFR via cell signaling cascades in AECs [37, 70, 71]. Production of IL-8 in BCG-infected human epithelial cells could be reduced by IL4, another important cytokine in human TB. However, IL-10 production was unaltered by IL- 4 in the AECs [72]. The expression of IL-10 was induced by IL-27 in bronchial epithelial cells regulated by the activation of the phosphatidylinositol 3-OH kinase (PI3K)-Akt signaling pathway [73]. Function of IL-27 was related to the pathogenesis of chronic obstructive pulmonary disease (COPD) and patients with pulmonary TB.

IL-18 is a member of the IL-1 cytokine superfamily and is secreted by macrophages and other cell types including human ATII cells. IL-18 plays an important role in the induction of a cell-mediated immunity by binding to its receptor following infection [74]. Human ATII cells are a
TABLE 2: Epithelial cell produced chemokines and cytokines against mycobacteria infections.

\begin{tabular}{llc}
\hline Inflammatory factor & Producing cells & Reference \\
\hline TNF- $\alpha$ & Alveolar epithelial cells & {$[9,65]$} \\
IFN- $\gamma$ & Alveolar epithelial cells & {$[66]$} \\
GM-CSF & Bronchial epithelial cells & {$[105]$} \\
IL-1 & Airway epithelial cells & {$[76]$} \\
IL-4 & Human bronchial epithelial & {$[72]$} \\
IL-6 & cells & {$[65]$} \\
IL-8 (CXCL8) & Alveolar epithelial cells & Airway epithelial cells, \\
IL-10 & alveolar epithelial cells & {$[65,66,76]$} \\
IL-18 & Bronchial epithelial cells & {$[73]$} \\
IL-27 & Human alveolar type II cells & {$[74,75]$} \\
IP-10 & Bronchial epithelial cells & {$[73]$} \\
MIG & Alveolar epithelial cells, & {$[67,76-78]$} \\
I-TAC & bronchial epithelial cells & {$[78]$} \\
MCP-1 & Bronchoepithelial cells & {$[78]$} \\
\hline
\end{tabular}

IP-10: interferon-inducible protein-10; MIG: monokine induced by IFNgamma; I-TAC: IFN-inducible T-cell alpha-chemoattractant; MCP-1: monocyte chemoattractant protein-1.

major resource of IL-18, and a baseline of IL-18 expression could be detected in normal ATII cells. The ATII cells and ATII-derived IL-18 might play a potential role in the pathomechanism of granulomatous in pulmonary TB. This is supported by the previous findings that ATII cells produce IL-18 in primary lung tissue cultures from patients with pulmonary TB [75]. This study found markedly increased IL-18 mRNA levels and increasing amounts of intracellular IL-18 protein with increasing culture time in ATII cell cultures stimulated with Mtb whole cell lysate. Additionally, the expression and secretion of mature IL-18 protein from human ATII cells was altered by the proinflammatory cytokines TNF- $\alpha$, IL- $1 \beta$, and IFN- $\gamma$ [75].

Other inflammatory factors found to be produced in mycobacteria infected airway epithelial cells include granulocyte colony-stimulating factor (G-CSF), GMCSF, interferon-inducible protein-10 (IP-10, also known as C-X-C-motif chemokine 10 (CXCL10), secreted by cells in response to IFN- $\gamma$ ), IFN- $\gamma$-inducible T-cell $\alpha$ chemoattractant (I-TAC, also called CXCL11 or IP-9, a small chemokine chemotactic for activated $\mathrm{T}$ cells), monokine induced by IFN- $\gamma$ (MIG, also known as CXCL9, a T-cell chemoattractant induced by IFN- $\gamma$ ), monocyte chemoattractant protein-1 (MCP-1), and leukotriene B4. The epithelial cells also produced inflammatory factors that play important roles in the recruitment of activated $\mathrm{T}$ cells and pathogenesis of pulmonary diseases in which IFN- $\gamma$ is highly expressed, such as pulmonary TB [67, 76-78].

3.3. Alveolar Epithelial Cell Necrosis and Mycobacterial Infection. Mycobacteria are able to invade and replicate in both 
macrophages and epithelial cells in the alveolar spaces of the lung. The alveolar epithelial cells actively contribute to the innate immune response in the lung and play an important role in mycobacterial dissemination during primary infection by undergoing cellular necrosis and releasing mycobacteria. Though the mycobacteria infection induces alveolar epithelial cell apoptosis, necrosis is the primary cause of cell death in infected alveolar cells. The necrosis was not a consequence of mycobacterial growth or the production of inflammatory factors in the host cells, but due to increased permeation of the cell membrane and the infection of live bacilli $[79,80]$. The suppressed apoptosis in the infected alveolar cells correlated with an upregulation of apoptosis inhibitors bcl-2 and Rb, and a downregulation of proapoptotic genes, BAD and BAX. This finding was opposite to that in mycobacteria-infected macrophages, suggesting that differential induction of apoptosis between macrophages and alveolar epithelial cells exists representing cell-specific strategies used by Mtb for survival in the host [80].

3.4. Epithelial Cells Secreted Matrix Metalloproteases and TB Immunopathology. The mechanism of Mtb lung tissue damage and mycobacterial spread remains poorly understood. Previous studies demonstrate that matrix metalloproteases (MMPs) have a unique ability to degrade fibrillar collagen at neutral $\mathrm{pH}$ leading to lung matrix destruction [81-85]. MMP activity was dependent upon a monocyteepithelial cell network and p38 MAPK phosphorylation to achieve the matrix-degrading phenotype [83]. The Mtb $6 \mathrm{kD}$ early secreted antigenic target (ESAT-6) protein was able to induce MMP-9 secretion from the epithelial cells neighboring infected macrophages [86]. Human primary bronchial epithelial cells cultured with conditioned medium from Mtb-infected monocytes (CoMTb) had upregulated levels of MMP-1. This increasing of MMP-1 was mediated via phosphorylation of p38 MAPK that was induced by synergism of CoMTb-driving TNF- $\beta$ and G protein-coupled receptor activation, and the decreasing of tissue inhibitor of metalloproteinase 1 (TIMP-1) secretion. Clinically it has been shown that activated p38 localized to the MMP-1secreting AECs of TB patients [81]. Furthermore, CoMTb was able to upregulate MMP-9 gene expression and secretion in primary human bronchial epithelial cells, while inhibition of the p38 MAPK activity led to decreased secretion of MMP-9. TNF- $\alpha$ was necessary, but not sufficient for MMP9 upregulation by a monocyte-epithelial cell network. This suggests that undefined soluble factors in CoMTb may synergize with TNF- $\alpha$ to increase MMP-9 secretion in the epithelial cells [82]. These results clearly imply that hostand pathogen-derived factors may work in concert to $\mathrm{Mtb}$ infection to drive MAPK-dependent MMPs secretion from AECs. Increased MMP secretion may lead to enhanced recruitment of macrophages, which contributed to earlier granuloma maturation and bacterial growth. This notion was supported by the fact that disruption of MMP9 function alleviated granuloma formation and bacterial growth [87]. Hence, interruption of the production of epithelial MMPs may be a therapeutic target for treatment of TB.
3.5. Airway Epithelial Cells Produce Nitric Oxide against Mycobacteria Infection. Nitric oxide (NO) and reactive oxygen intermediates are toxic molecules used by the immune system. Production of NO by host cells is an effective host defense mechanism against microbial infection. Bose et al. demonstrated that in a murine TB model NO production played an essential role in Mtb killing by mononuclear phagocytes, particularly during the early phase of the infection. Additionally, it may also play a role in tissue damage during the late phases of the disease [88]. In vitro studies using A549 cells showed an induction of NO in response to Mtb infection, which is consistent with the finding that NO has antimicrobial effects against Mtb infection [89]. These results suggest that a correlation exists between NO production and innate immunity of AECs against mycobacteria [90].

\section{Airway Epithelial Cells: The Connection of Innate and Adaptive Immunity}

Airway epithelial cells have been recognized to play important roles in the defense of invaded microbial pathogens, via both the innate and adaptive immune responses [2, 4]. It has become increasingly evident that AECs express PRRs that recognize microbial pathogens and activate innate host defense mechanisms in the airway. Furthermore, the activated innate response secondarily induces recruitment and activation of DCs, T cells, and B cells that augment antigen recognition, antibody production, and other adaptive immune components. These response mechanisms include epithelial production of cytokines and chemokines that stimulate communication between the "nonprofessional" (epithelial cells) and "professional" (B cells, T cells, DCs, and macrophages) immune cells [4].

Following infection of mycobacteria, the host immune system must first recognize the invading pathogens, activate the innate response, and initiate the adaptive immune response. Though "professional" immune cells play a central role in the initiation of adaptive immunity, the alveolar epithelial cells are among the first cell types to encounter mycobacterial pathogens, implying that crosstalk between alveolar epithelial cells and "professional" immune cells may be important in initiating a coordinated secretion of chemokines and the subsequent recruitment of leukocytes to the lung. ATII cells are capable of producing various antimicrobial and proinflammatory molecules that contribute to pulmonary immunity. These cells do so not only by secreting chemokines that recruit inflammatory cells to the lung but also serving as antigen-presenting cells [91]. Although ATII cells are unlikely to prime naïve $\mathrm{T}$ cells, their ability to present antigens to $\mathrm{T}$ cells suggest that they may play a role in the effector phase of the immune response [91]. Interestingly, the migration of monocytes across the alveolar epithelial-endothelial barrier required the production of chemokines and the presence of surface molecules on both the alveolar epithelial and endothelial cells [10]. These studies demonstrate a novel role for ATII cells in the immunological response to pulmonary pathogens such 
as Mtb [91]. However, the interaction between AECs and immune cells (e.g., the macrophages) in response to $\mathrm{Mtb}$ infection is currently largely unknown.

\section{Concluding Remarks}

It is well accepted that airway epithelium plays an important role in the innate immune response by its collection of surface, endosomal, and cytosolic sensors that activate numerous proinflammatory signal pathways. Additionally, resident antimicrobial substances secreted by the epithelial cells offer significant mechanisms to deal with the invaded pathogens. A growing body of evidence supports the essential involvement of AECs in both the innate and adaptive immune responses against mycobacterial infection. These studies have facilitated the understanding of the major components of the innate immune system and their role in the host's defense of AECs, despite the fact that the interaction between AECs and Mtb is far less known in comparison with the interplay of alveolar macrophages and this pathogen. A recent study using Mycobacterium marinum in the zebra fish model revealed a molecular mechanism of mycobacteria induce granulomas, a hallmark of tuberculosis. In this mechanism model, the mycobacteria secreted ESAT6 induced MMP-9 in those epithelial cells neighboring the infected macrophages, which in turn contributed to nascent granuloma maturation and bacterial growth by increasing the recruitment of macrophages to sites of infection $[86,87]$. Together with the findings of the ability of epithelial cellsecreted SPs to regulate the initial interaction between Mtb and alveolar macrophage, these studies provide an insight into the interaction/crosstalk between macrophages and AEC in pathogenesis of TB $[48,49,86,87]$.

AECs act to sense infectious danger by functionally expressing PRRs. Meanwhile, they are able to limit potentially harmful inflammatory reactions within the framework of organ-specific immunity by increasing the activation threshold [92]. In the case of mycobacterial infection, interaction between the pathogen and AECs results in the production of antimicrobial substances or peptides, lysozyme, lactoferrin, $\beta$-defensins, and NO. Furthermore, these cells produce and secrete proinflammatory cytokines and chemokines thus initiating the adaptive response by recruitment of inflammatory cells. The mechanisms used by the airway epithelium to discriminate between pathogenic or nonpathogenic Mycobacterium, sense pathogens to immune effectors, interaction/crosstalk between AECs and alveolar macrophages, as well as the clinical relevance of epithelial cell-mediated immune responses, have yet to be fully elucidated.

\section{Conflict of Interests}

The authors declare that there are no conflict of interests.

\section{Acknowledgments}

This work was supported by a grant for the National Basic Science Program of China (973 Program) (2012CB518801) and grants (nos. 31172278 and 31160515) from the National Natural Science Foundation of China.

\section{References}

[1] R. Bals and P. S. Hiemstra, "Innate immunity in the lung: how epithelial cells fight against respiratory pathogens," European Respiratory Journal, vol. 23, no. 2, pp. 327-333, 2004.

[2] A. Kato and R. P. Schleimer, "Beyond inflammation: airway epithelial cells are at the interface of innate and adaptive immunity," Current Opinion in Immunology, vol. 19, no. 6, pp. 711-720, 2007.

[3] T. S. McCormick and A. Weinberg, "Epithelial cell-derived antimicrobial peptides are multifunctional agents that bridge innate and adaptive immunity," Periodontology 2000, vol. 54, no. 1, pp. 195-206, 2010.

[4] J. H. Ryu, C. H. Kim, and J. H. Yoon, "Innate immune responses of the airway epithelium," Molecules and Cells, vol. 30, no. 3, pp. 173-183, 2010.

[5] A. G. Tsolaki, "Innate immune recognition in tuberculosis infection," Advances in Experimental Medicine and Biology, vol. 653, pp. 185-197, 2009.

[6] L. E. Bermudez and J. Goodman, "Mycobacterium tuberculosis invades and replicates within type II alveolar cells," Infection and Immunity, vol. 64, no. 4, pp. 1400-1406, 1996.

[7] R. Teitelbaum, W. Schubert, L. Gunther et al., "The M cell as a portal of entry to the lung for the bacterial pathogen Mycobacterium tuberculosis," Immunity, vol. 10, no. 6, pp. 641-650, 1999.

[8] K. A. McDonough and Y. Kress, "Cytotoxicity for lung epithelial cells is a virulence-associated phenotype of Mycobacterium tuberculosis," Infection and Immunity, vol. 63, no. 12, pp. 4802-4811, 1995.

[9] M. Zhang, K. J. Kim, D. Iyer et al., "Effects of Mycobacterium tuberculosis on the bioelectric properties of the alveolar epithelium," Infection and Immunity, vol. 65, no. 2, pp. 692698, 1997.

[10] L. E. Bermudez, F. J. Sangari, P. Kolonoski, M. Petrofsky, and J. Goodman, "The efficiency of the translocation of Mycobacterium tuberculosis across a bilayer of epithelial and endothelial cells as a model of the alveolar wall is a consequence of transport within mononuclear phagocytes and invasion of alveolar epithelial cells," Infection and Immunity, vol. 70, no. 1, pp. 140-146, 2002.

[11] L. Hall-Stoodley, G. Watts, J. E. Crowther et al., "Mycobactenum tuberculosis binding to human surfactant proteins $\mathrm{A}$ and $\mathrm{D}$, fibronectin, and small airway epithelial cells under shear conditions," Infection and Immunity, vol. 74, no. 6, pp. 3587-3596, 2006.

[12] R. van Crevel, J. Kleinnijenhuis, M. Oosting, L. A. B. Joosten, and M. G. Netea, "Innate immune recognition of Mycobacterium tuberculosis," Clinical and Developmental Immunology, vol. 2011, Article ID 405310, 12 pages, 2011.

[13] A. K. Mayer, M. Muehmer, J. Mages et al., "Differential recognition of TLR-dependent microbial ligands in human bronchial epithelial cells," The Journal of Immunology, vol. 178, no. 5, pp. 3134-3142, 2007.

[14] J. S. Ferguson and L. S. Schlesinger, "Pulmonary surfactant in innate immunity and the pathogenesis of tuberculosis," Tubercle and Lung Disease, vol. 80, no. 4-5, pp. 173-184, 2000.

[15] X. Ma, Y. Liu, B. B. Gowen, E. A. Graviss, A. G. Clark, and J. M. Musser, "Full-exon resequencing reveals Toll-like receptor 
variants contribute to human susceptibility to tuberculosis disease," PLoS One, vol. 2, no. 12, Article ID e1318, 2007.

[16] A. G. Bowie, "Translational mini-review series on Tolllike receptors: recent advances in understanding the role of Toll-like receptors in anti-viral immunity," Clinical and Experimental Immunology, vol. 147, no. 2, pp. 217-226, 2007.

[17] K. Heeg, A. Dalpke, M. Peter, and S. Zimmermann, "Structural requirements for uptake and recognition of $\mathrm{CpG}$ oligonucleotides," International Journal of Medical Microbiology, vol. 298, no. 1-2, pp. 33-38, 2008.

[18] Q. Sha, A. Q. Truong-Tran, J. R. Plitt, L. A. Beck, and R. P. Schleimer, "Activation of airway epithelial cells by Toll-like receptor agonists," American Journal of Respiratory Cell and Molecular Biology, vol. 31, no. 3, pp. 358-364, 2004.

[19] C. M. Fremond, V. Yeremeev, D. M. Nicolle, M. Jacobs, V. F. Quesniaux, and B. Ryffel, "Fatal Mycobacterium tuberculosis infection despite adaptive immune response in the absence of MyD88," Journal of Clinical Investigation, vol. 114, no. 12, pp. 1790-1799, 2004.

[20] G. Diamond, D. Legarda, and L. K. Ryan, "The innate immune response of the respiratory epithelium," Immunological Reviews, vol. 173, pp. 27-38, 2000.

[21] J. E. Petrikin, R. Gaedigk, J. S. Leeder, and W. E. Truog, "Selective Toll-like receptor expression in human fetal lung," Pediatric Research, vol. 68, no. 4, pp. 335-338, 2010.

[22] A. O. Aliprantis, R. B. Yang, M. R. Mark et al., "Cell activation and apoptosis by bacterial lipoproteins through Toll- like receptor-2," Science, vol. 285, no. 5428, pp. 736-739, 1999.

[23] O. Takeuchi, S. Sato, T. Horiuchi et al., "Cutting edge: role of Toll-like receptor 1 in mediating immune response to microbial lipoproteins," The Journal of Immunology, vol. 169, no. 1, pp. 10-14, 2002.

[24] K. Farhat, S. Riekenberg, H. Heine et al., "Heterodimerization of TLR2 with TLR1 or TLR6 expands the ligand spectrum but does not lead to differential signaling," Journal of Leukocyte Biology, vol. 83, no. 3, pp. 692-701, 2008.

[25] P. Uciechowski, H. Imhoff, C. Lange et al., "Susceptibility to tuberculosis is associated with TLR1 polymorphisms resulting in a lack of TLR1 cell surface expression," Journal of Leukocyte Biology, vol. 90, no. 2, pp. 377-388, 2011.

[26] M. S. Jin, S. E. Kim, J. Y. Heo et al., "Crystal structure of the TLR1-TLR2 heterodimer induced by binding of a tri-acylated lipopeptide," Cell, vol. 130, no. 6, pp. 1071-1082, 2007.

[27] M. B. Drennan, D. Nicolle, V. J. F. Quesniaux et al., “TollLike receptor 2-deficient mice succumb to Mycobacterium tuberculosis infection," American Journal of Pathology, vol. 164, no. 1, pp. 49-57, 2004.

[28] A. Biragyn, P. A. Ruffini, C. A. Leifer et al., "Toll-like receptor 4 -dependent activation of dendritic cells by $\beta$-defensin 2 ," Science, vol. 298, no. 5595, pp. 1025-1029, 2002.

[29] D. Yang, O. Chertov, S. N. Bykovskaia et al., “ $\beta$-Defensins: linking innate and adaptive immunity through dendritic and T cell CCR6," Science, vol. 286, no. 5439, pp. 525-528, 1999.

[30] C. J. Hertz, Q. Wu, E. M. Porter et al., "Activation of Tolllike receptor 2 on human tracheobronchial epithelial cells induces the antimicrobial peptide human $\beta$ defensin-2," The Journal of Immunology, vol. 171, no. 12, pp. 6820-6826, 2003.

[31] L. Guillott, S. Medjane, K. Le-Barillec et al., "Response of human pulmonary epithelial cells to lipopolysaccharide involves Toll-like receptor 4 (TLR4)-dependent signaling pathways: evidence for an intracellular compartmentalization of TLR4," The Journal of Biological Chemistry, vol. 279, no. 4, pp. 2712-2718, 2004.
[32] C. Schulz, L. Farkas, K. Wolf, K. Kratzel, G. Eissner, and M. Pfeifer, "Differences in LPS-induced activation of bronchial epithelial cells (BEAS-2B) and type II-like pneumocytes (A549)," Scandinavian Journal of Immunology, vol. 56, no. 3, pp. 294-302, 2002.

[33] J. Branger, J. C. Leemans, S. Florquin, S. Weijer, P. Speelman, and T. van der Poll, "Toll-like receptor 4 plays a protective role in pulmonary tuberculosis in mice," International Immunology, vol. 16, no. 3, pp. 509-516, 2004.

[34] B. Abel, N. Thieblemont, V. J. F. Quesniaux et al., "Tolllike receptor 4 expression is required to control chronic Mycobacterium tuberculosis infection in mice," The Journal of Immunology, vol. 169, no. 6, pp. 3155-3162, 2002.

[35] N. Reiling, C. Holscher, A. Fehrenbach et al., "Cutting edge: Toll-like receptor (TLR)2- and TLR4-mediated pathogen recognition in resistance to airborne infection with Mycobacterium tuberculosis," The Journal of Immunology, vol. 169, no. 7, pp. 3480-3484, 2002.

[36] J. M. Duggan, D. You, J. O. Cleaver et al., "Synergistic interactions of TLR2/6 and TLR9 induce a high level of resistance to lung infection in mice," The Journal of Immunology, vol. 186, no. 10, pp. 5916-5926, 2011.

[37] J. L. Koff, M. X. G. Shao, I. F. Ueki, and J. A. Nadel, "Multiple TLRs activate EGFR via a signaling cascade to produce innate immune responses in airway epithelium," American Journal of Physiology, vol. 294, no. 6, pp. L1068-L1075, 2008.

[38] A. G. Rothfuchs, A. Bafica, C. G. Feng et al., "Dectin-1 interaction with Mycobacterium tuberculosis leads to enhanced IL12 p40 production by splenic dendritic cells," The Journal of Immunology, vol. 179, no. 6, pp. 3463-3471, 2007.

[39] H. M. Lee, J. M. Yuk, D. M. Shin, and E. K. Jo, "Dectin1 is inducible and plays an essential role for mycobacteriainduced innate immune responses in airway epithelial cells," Journal of Clinical Immunology, vol. 29, no. 6, pp. 795-805, 2009.

[40] F. L. van de Veerdonk, A. C. Teirlinck, J. Kleinnijenhuis et al., "Mycobacterium tuberculosis induces IL-17A responses through TLR4 and dectin-1 and is critically dependent on endogenous IL-1," Journal of Leukocyte Biology, vol. 88, no. 2, pp. 227-232, 2010.

[41] S. T. Liffers, J. B. Munding, M. Vogt et al., "MicroRNA$148 \mathrm{a}$ is down-regulated in human pancreatic ductal adenocarcinomas and regulates cell survival by targeting CDC25B," Laboratory Investigation, vol. 91, no. 10, pp. 1472-1479, 2011.

[42] J. R. Wright, "Immunoregulatory functions of surfactant proteins," Nature Reviews Immunology, vol. 5, no. 1, pp. 5868, 2005.

[43] X. Xu, K. McCormick-Shannon, D. R. Voelker, and R. J. Mason, "KGF increases SP-A and SP-D mRNA levels and secretion in cultured rat alveolar type II cells," American Journal of Respiratory Cell and Molecular Biology, vol. 18, no. 2, pp. 168-178, 1998.

[44] M. P. Lemos, J. McKinney, and K. Y. Rhee, "Dispensability of surfactant proteins A and D in immune control of Mycobacterium tuberculosis infection following aerosol challenge of mice," Infection and Immunity, vol. 79, no. 3, pp. 1077-1085, 2011.

[45] Z. C. Chroneos, K. Midde, Z. Sever-Chroneos, and C. Jagannath, "Pulmonary surfactant and tuberculosis," Tuberculosis, vol. 89, supplement 1, pp. S10-S14, 2009.

[46] R. Haataja and M. Hallman, "Surfactant proteins as genetic determinants of multifactorial pulmonary diseases," Annals of Medicine, vol. 34, no. 5, pp. 324-333, 2002. 
[47] U. Kishore, A. L. Bernal, M. F. Kamran et al., "Surfactant proteins SP-A and SP-D in human health and disease," Archivum Immunologiae et Therapiae Experimentalis, vol. 53, no. 5, pp. 399-417, 2005.

[48] C. D. Gaynor, F. X. McCormack, D. R. Voelker, S. E. McGowan, and L. S. Schlesinger, "Pulmonary surfactant protein A mediates enhanced phagocytosis of Mycobacterium tuberculosis by a direct interaction with human macrophages," The Journal of Immunology, vol. 155, no. 11, pp. 5343-5351, 1995.

[49] S. Sidobre, J. Nigou, G. Puzo, and M. Riviere, "Lipoglycans are putative ligands for the human pulmonary surfactant protein A attachment to mycobacteria: critical role of the lipids for lectin- carbohydrate recognition," The Journal of Biological Chemistry, vol. 275, no. 4, pp. 2415-2422, 2000.

[50] L. F. Weikert, J. P. Lopez, R. Abdolrasulnia, Z. C. Chroneos, and V. L. Shepherd, "Surfactant protein A enhances mycobacterial killing by rat macrophages through a nitric oxide-dependent pathway," American Journal of Physiology, vol. 279, no. 2, pp. L216-L223, 2000.

[51] A. Ragas, L. Roussel, G. Puzo, and M. Riviere, "The Mycobacterium tuberculosis cell-surface glycoprotein apa as a potential adhesin to colonize target cells via the innate immune system pulmonary C-type lectin surfactant protein A," The Journal of Biological Chemistry, vol. 282, no. 8, pp. 5133-5142, 2007.

[52] T. K. Carlson, J. B. Torrelles, K. Smith et al., "Critical role of amino acid position 343 of surfactant protein-D in the selective binding of glycolipids from Mycobacterium tuberculosis," Glycobiology, vol. 19, no. 12, pp. 1473-1484, 2009.

[53] J. S. Ferguson, D. R. Voelker, J. A. Ufnar, A. J. Dawson, and L. S. Schlesinger, "Surfactant protein D inhibition of human macrophage uptake of Mycobacterium tuberculosis is independent of bacterial agglutination," The Journal of Immunology, vol. 168, no. 3, pp. 1309-1314, 2002.

[54] J. S. Ferguson, J. L. Martin, A. K. Azad et al., "Surfactant protein D increases fusion of Mycobacterium tuberculosis- containing phagosomes with lysosomes in human macrophages," Infection and Immunity, vol. 74, no. 12, pp. 7005-7009, 2006.

[55] B. Rivas-Santiago, R. Hernandez-Pando, C. Carranza et al., "Expression of cathelicidin LL-37 during Mycobacterium tuberculosis infection in human alveolar macrophages, monocytes, neutrophils, and epithelial cells," Infection and Immunity, vol. 76, no. 3, pp. 935-941, 2008.

[56] P. Mendez-Samperio, A. Perez, and L. Torres, "Role of reactive oxygen species (ROS) in Mycobacterium bovis bacillus Calmette Guerin-mediated up-regulation of the human cathelicidin LL-37 in A549 cells," Microbial Pathogenesis, vol. 47, no. 5, pp. 252-257, 2009.

[57] P. Mendez-Samperio, E. Miranda, and A. Trejo, "Expression and secretion of cathelicidin LL-37 in human epithelial cells after infection by Mycobacterium bovis bacillus CalmetteGuerin," Clinical and Vaccine Immunology, vol. 15, no. 9, pp. 1450-1455, 2008.

[58] B. Rivas-Santiago, S. K. Schwander, C. Sarabia et al., "Human $\beta$-defensin 2 is expressed and associated with Mycobacterium tuberculosis during infection of human alveolar epithelial cells," Infection and Immunity, vol. 73, no. 8, pp. 4505-4511, 2005.

[59] P. Mendez-Samperio, E. Miranda, and A. Trejo, "Mycobacterium bovis Bacillus Calmette-Guerin (BCG) stimulates human $\beta$-defensin-2 gene transcription in human epithelial cells," Cellular Immunology, vol. 239, no. 1, pp. 61-66, 2006.

[60] F. B. Sow, S. Nandakumar, V. Velu et al., "Mycobacterium tuberculosis components stimulate production of the antimicrobial peptide hepcidin," Tuberculosis, vol. 91, no. 4, pp. 314-321, 2011.

[61] P. Strnad, P. Schwarz, M. C. D. Rasenack et al., "Hepcidin is an antibacterial, stress-inducible peptide of the biliary system," PLoS One, vol. 6, no. 1, Article ID e16454, 2011.

[62] M. I. Gomez and A. Prince, "Airway epithelial cell signaling in response to bacterial pathogens," Pediatric Pulmonology, vol. 43, no. 1, pp. 11-19, 2008.

[63] A. M. Cooper and S. A. Khader, "The role of cytokines in the initiation, expansion, and control of cellular immunity to tuberculosis," Immunological Reviews, vol. 226, no. 1, pp. 191-204, 2008.

[64] W. Peters and J. D. Ernst, "Mechanisms of cell recruitment in the immune response to Mycobacterium tuberculosis," Microbes and Infection, vol. 5, no. 2, pp. 151-158, 2003.

[65] H. M. Lee, D. M. Shin, and E. K. Jo, "Mycobacterium tuberculosis induces the production of tumor necrosis factor- $\alpha$, interleukin-6, and CXCL8 in pulmonary epithelial cells through reactive oxygen species-dependent mitogenactivated protein kinase activation," Journal Bacteriology and Virology, vol. 39, no. 1, pp. 1-10, 2009.

[66] M. Sharma, S. Sharma, S. Roy, S. Varma, and M. Bose, "Pulmonary epithelial cells are a source of interferon- $\gamma$ in response to Mycobacterium tuberculosis infection," Immunology and Cell Biology, vol. 85, no. 3, pp. 229-237, 2007.

[67] Y. Lin, M. Zhang, and P. F. Barnes, "Chemokine production by a human alveolar epithelial cell line in response to Mycobacterium tuberculosis," Infection and Immunity, vol. 66, no. 3, pp. 1121-1126, 1998.

[68] J. S. Park, Y. S. Kim, Y. K. Jee, N. H. Myong, and K. Y. Lee, "Interleukin-8 production in tuberculous pleurisy: role of mesothelial cells stimulated by cytokine network involving tumour necrosis factor- $\alpha$ and interleukin- $1 \beta$," Scandinavian Journal of Immunology, vol. 57, no. 5, pp. 463-469, 2003.

[69] T. Nakanaga, J. A. Nadel, I. F. Ueki, J. L. Koff, and M. X. G. Shao, "Regulation of interleukin-8 via an airway epithelial signaling cascade," American Journal of Physiology, vol. 292, no. 5, pp. L1289-L1296, 2007.

[70] R. Sorrentino, P. M. de Souza, S. Sriskandan, C. Duffin, M. J. Paul-Clark, and J. A. Mitchell, "Pattern recognition receptors and interleukin-8 mediate effects of Gram-positive and Gram-negative bacteria on lung epithelial cell function," British Journal of Pharmacology, vol. 154, no. 4, pp. 864-871, 2008.

[71] P. Mendez-Samperio, L. Belmont, and E. Miranda, "Mycobacterium bovis BCG Toll-like receptors 2 and 4 cooperation increases the innate epithelial immune response," Archives of Medical Research, vol. 39, no. 1, pp. 33-39, 2008.

[72] P. Mendez-Samperio, E. Miranda, and A. Vazquez, "Expression and secretion of CXCL-8 and CXCL-10 from Mycobacterium bovis BCG-infected human epithelial cells: role of IL4," Mediators of Inflammation, vol. 2006, Article ID 67451, 2006.

[73] J. Cao, Z. Liping, L. Dairong et al., "IL-27 is elevated in COPD and PTB patients and induces human bronchial epithelial cells to produce CXCL10," Chest, vol. 141, no. 1, pp. 121-130, 2012.

[74] Z. Liu, H. Wang, W. Xiao, C. Wang, G. Liu, and T. Hong, "Thyrocyte interleukin-18 expression is up-regulated by 
interferon- $\gamma$ and may contribute to thyroid destruction in Hashimoto's thyroiditis," International Journal of Experimental Pathology, vol. 91, no. 5, pp. 420-425, 2010.

[75] D. V. Pechkovsky, T. Goldmann, E. Vollmer, J. MullerQuernheim, and G. Zissel, "Interleukin-18 expression by alveolar epithelial cells type II in tuberculosis and sarcoidosis," FEMS Immunology and Medical Microbiology, vol. 46, no. 1, pp. 30-38, 2006.

[76] M. I. Wickremasinghe, L. H. Thomas, and J. S. Friedland, "Pulmonary epithelial cells are a source of IL- 8 in the response to Mycobacterium tuberculosis: essential role of IL1 from infected monocytes in a NF- $\kappa \mathrm{B}$-dependent network," The Journal of Immunology, vol. 163, no. 7, pp. 3936-3947, 1999.

[77] K. Sato, H. Tomioka, T. Shimizu, T. Gonda, F. Ota, and C. Sano, "Type II alveolar cells play roles in macrophagemediated host innate resistance to pulmonary mycobacterial infections by producing proinflammatory cytokines," Journal of Infectious Diseases, vol. 185, no. 8, pp. 1139-1147, 2002.

[78] A. Sauty, M. Dziejman, R. A. Taha et al., "The T cell-specific CXC chemokines IP-10, Mig, and I-TAC are expressed by activated human bronchial epithelial cells," The Journal of Immunology, vol. 162, no. 6, pp. 3549-3558, 1999.

[79] K. M. Dobos, E. A. Spotts, F. D. Quinn, and C. H. King, "Necrosis of lung epithelial cells during infection with Mycobacterium tuberculosis is preceded by cell permeation," Infection and Immunity, vol. 68, no. 11, pp. 6300-6310, 2000.

[80] L. Danelishvili, J. McGarvey, Y. J. Li, and L. E. Bermudez, "Mycobacterium tuberculosis infection causes different levels of apoptosis and necrosis in human macrophages and alveolar epithelial cells," Cellular Microbiology, vol. 5, no. 9, pp. 649-660, 2003.

[81] P. T. G. Elkington, J. E. Emerson, L. D. C. Lopez-Pascua et al., "Mycobacterium tuberculosis up-regulates matrix metalloproteinase-1 secretion from human airway epithelial cells via a p38 MAPK switch," The Journal of Immunology, vol. 175, no. 8, pp. 5333-5340, 2005.

[82] P. T. Elkington, J. A. Green, J. E. Emerson et al., "Synergistic up-regulation of epithelial cell matrix metalloprotemase-9 secretion in tuberculosis," American Journal of Respiratory Cell and Molecular Biology, vol. 37, no. 4, pp. 431-437, 2007.

[83] P. T. Elkington, C. A. Ugarte-Gil, and J. S. Friedland, "Matrix metalloproteinases in tuberculosis," European Respiratory Journal, vol. 38, no. 2, pp. 456-464, 2011.

[84] S. P. Anand and P. Selvaraj, "Effect of 1,25 dihydroxyvitamin D3 on matrix metalloproteinases MMP-7, MMP-9 and the inhibitor TIMP-1 in pulmonary tuberculosis," Clinical Immunology, vol. 133, no. 1, pp. 126-131, 2009.

[85] S. El-Masry, M. Lotfy, M. Samy, S. Moawia, I. El-Sayed, and I. Khamees, "Pattern of matrix metalloproteinases-9, P53 and BCL-2 proteins in Egyptian patients with pulmonary Mycobacterium tuberculosis," Acta Microbiologica et Immunologica Hungarica, vol. 57, no. 2, pp. 123-133, 2010.

[86] K. Aoki, S. Matsumoto, Y. Hirayama et al., "Extracellular mycobacterial DNA-binding protein 1 participates in Mycobacterium-lung epithelial cell interaction through hyaluronic acid," The Journal of Biological Chemistry, vol. 279, no. 38, pp. 39798-39806, 2004.

[87] H. E. Volkman, T. C. Pozos, J. Zheng, J. M. Davis, J. F. Rawls, and L. Ramakrishnan, "Tuberculous granuloma induction via interaction of a bacterial secreted protein with host epithelium," Science, vol. 327, no. 5964, pp. 466-469, 2010.

[88] M. Bose, P. Farnia, S. Sharma, D. Chattopadhya, and K. Saha, "Nitric oxide dependent killing of Mycobacterium tuberculosis by human mononuclear phagocytes from patients with active tuberculosis," International Journal of Immunopathology and Pharmacology, vol. 12, no. 2, pp. 69-79, 1999.

[89] R. Ciccone, F. Mariani, A. Cavone et al., "Inhibitory effect of NO-releasing ciprofloxacin (NCX 976) on Mycobacterium tuberculosis survival," Antimicrobial Agents and Chemotherapy, vol. 47, no. 7, pp. 2299-2302, 2003.

[90] S. Roy, S. Sharma, M. Sharma, R. Aggarwal, and M. Bose, "Induction of nitric oxide release from the human alveolar epithelial cell line A549: an in vitro correlate of innate immune response to Mycobacterium tuberculosis," Immunology, vol. 112, no. 3, pp. 471-480, 2004.

[91] H. Debbabi, S. Ghosh, A. B. Kamath et al., "Primary type II alveolar epithelial cells present microbial antigens to antigenspecific CD4 ${ }^{+}$T cells," American Journal of Physiology, vol. 289, no. 2, pp. L274-L279, 2005.

[92] A. K. Mayer and A. H. Dalpke, "Regulation of local immunity by airway epithelial cells," Archivum Immunologiae et Therapiae Experimentalis, vol. 55, no. 6, pp. 353-362, 2007.

[93] A. K. Randhawa, M. S. Shey, A. Keyser et al., "Association of human TLR1 and TLR6 deficiency with altered immune responses to bcg vaccination in south african infants," PLoS Pathogens, vol. 7, no. 8, Article ID e1002174, 2011.

[94] M. A. Chambers, A. O. Whelan, R. Spallek et al., "Nonacylated Mycobacterium bovis glycoprotein MPB83 binds to TLR1/2 and stimulates production of matrix metalloproteinase 9," Biochemical and Biophysical Research Communications, vol. 400, no. 3, pp. 403-408, 2010.

[95] A. Uehara, Y. Fujimoto, K. Fukase, and H. Takada, "Various human epithelial cells express functional Toll-like receptors, NOD1 and NOD2 to produce anti-microbial peptides, but not proinflammatory cytokines," Molecular Immunology, vol. 44, no. 12, pp. 3100-3111, 2007.

[96] D. Torres, A. Dieudonne, B. Ryffel et al., "Double-stranded RNA exacerbates pulmonary allergic reaction through TLR3: implication of airway epithelium and dendritic cells," The Journal of Immunology, vol. 185, no. 1, pp. 451-459, 2010.

[97] T. Numata, J. Araya, S. Fujii et al., "Insulin-dependent phosphatidylinositol 3-kinase/Akt and ERK signaling pathways inhibit TLR3-mediated human bronchial epithelial cell apoptosis," The Journal of Immunology, vol. 187, no. 1, pp. 510-519, 2011.

[98] C. Zhang, X. Wu, Y. Zhao, Z. Deng, and G. Qian, "SIGIRR inhibits Toll-like receptor 4, 5, 9-mediated immune responses in human airway epithelial cells," Molecular Biology Reports, vol. 38, no. 1, pp. 601-609, 2011.

[99] H. Zhang, L. Liu, K. Wen et al., "Chimeric flagellin expressed by Salmonella typhimurium induces an ESAT-6-specific Th1type immune response and CTL effects following intranasal immunization," Cellular and Molecular Immunology, vol. 8, no. 6, pp. 496-501, 2011.

[100] S. Chatterjee, V. P. Dwivedi, Y. Singh et al., "Early secreted antigen ESAT-6 of Mycobacterium tuberculosis promotes protective $\mathrm{T}$ helper 17 cell responses in a Toll-like receptor2-dependent manner," PLoS Pathogens, vol. 7, no. 11, Article ID e1002378, 2011.

[101] M. P. Gantier, A. T. Irving, M. Kaparakis-Liaskos et al., "Genetic modulation of TLR8 response following bacterial phagocytosis," Human Mutation, vol. 31, no. 9, pp. 10691079, 2010.

[102] H. C. Lee and S. F. Ziegler, "Inducible expression of the proallergic cytokine thymic stromal lymphopoietin in airway epithelial cells is controlled by $\mathrm{NF} \kappa \mathrm{B}$," Proceedings of the 
National Academy of Sciences of the United States of America, vol. 104, no. 3, pp. 914-919, 2007.

[103] S. Davila, M. L. Hibberd, R. H. Dass et al., "Genetic association and expression studies indicate a role of Toll-like receptor 8 in pulmonary tuberculosis," PLoS Genetics, vol. 4, no. 10, Article ID e1000218, 2008.

[104] D. R. Velez, C. Wejse, M. E. Stryjewski et al., "Variants in Tolllike receptors 2 and 9 influence susceptibility to pulmonary tuberculosis in Caucasians, African-Americans, and West Africans," Human Genetics, vol. 127, no. 1, pp. 65-73, 2010.

[105] M. Marini, M. Soloperto, Y. Zheng, M. Mezzetti, and S. Mattoli, "Protective effect of nedocromil sodium on the IL1induced release of GM-CSF from cultured human bronchial epithelial cells," Pulmonary Pharmacology, vol. 5, no. 1, pp. 61-65, 1992. 


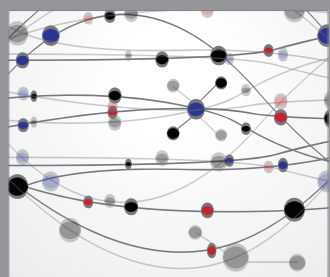

The Scientific World Journal
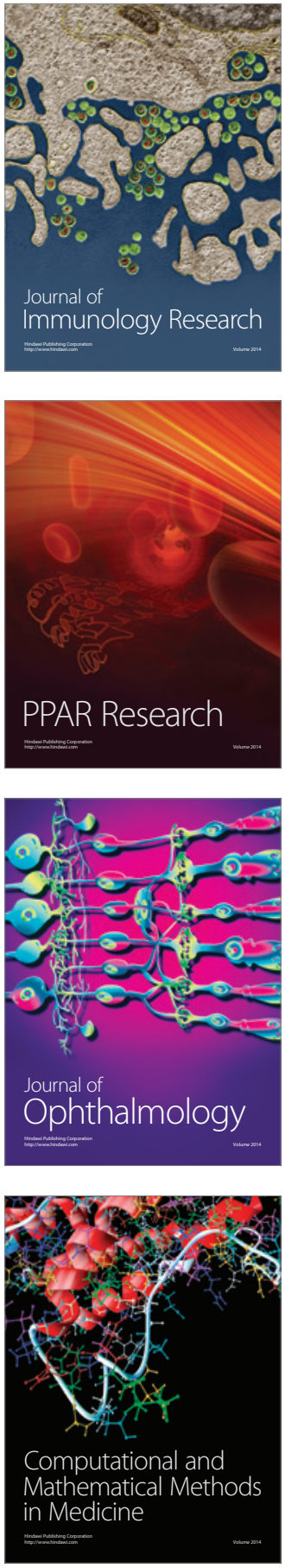

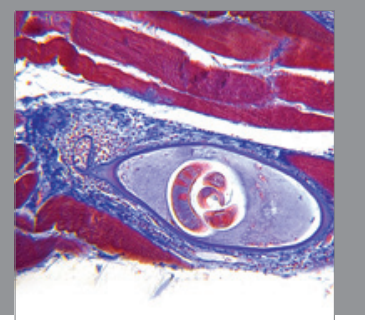

Gastroenterology

Research and Practice
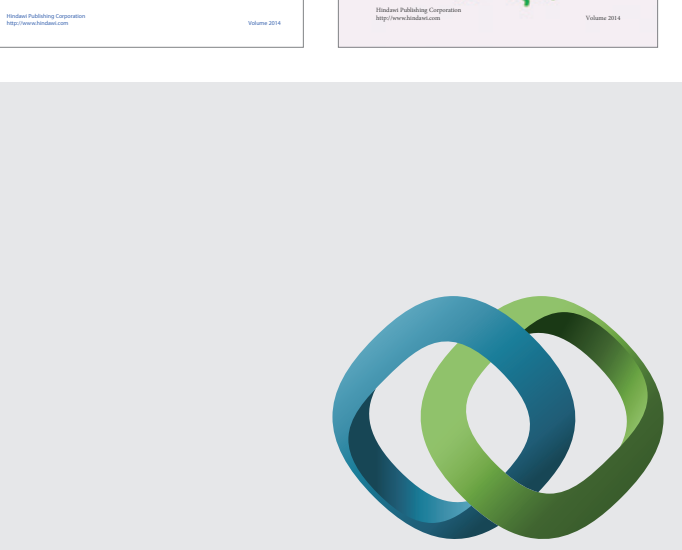

\section{Hindawi}

Submit your manuscripts at

http://www.hindawi.com
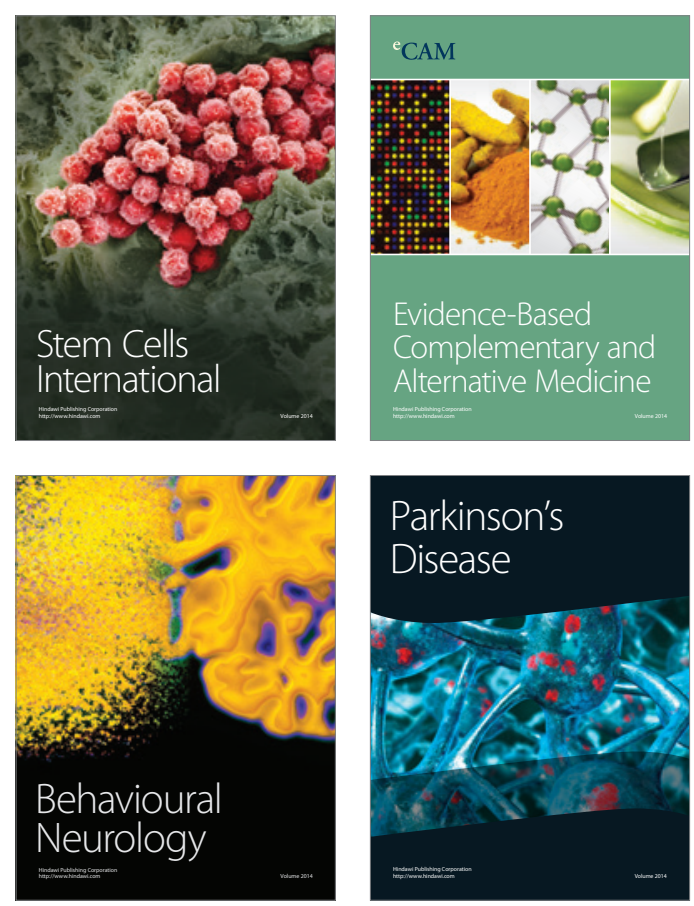

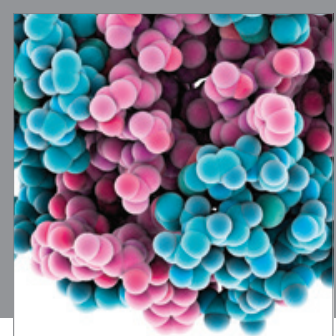

Journal of
Diabetes Research

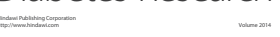

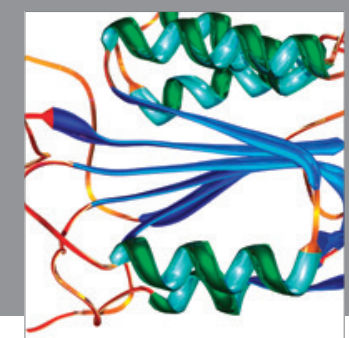

Disease Markers
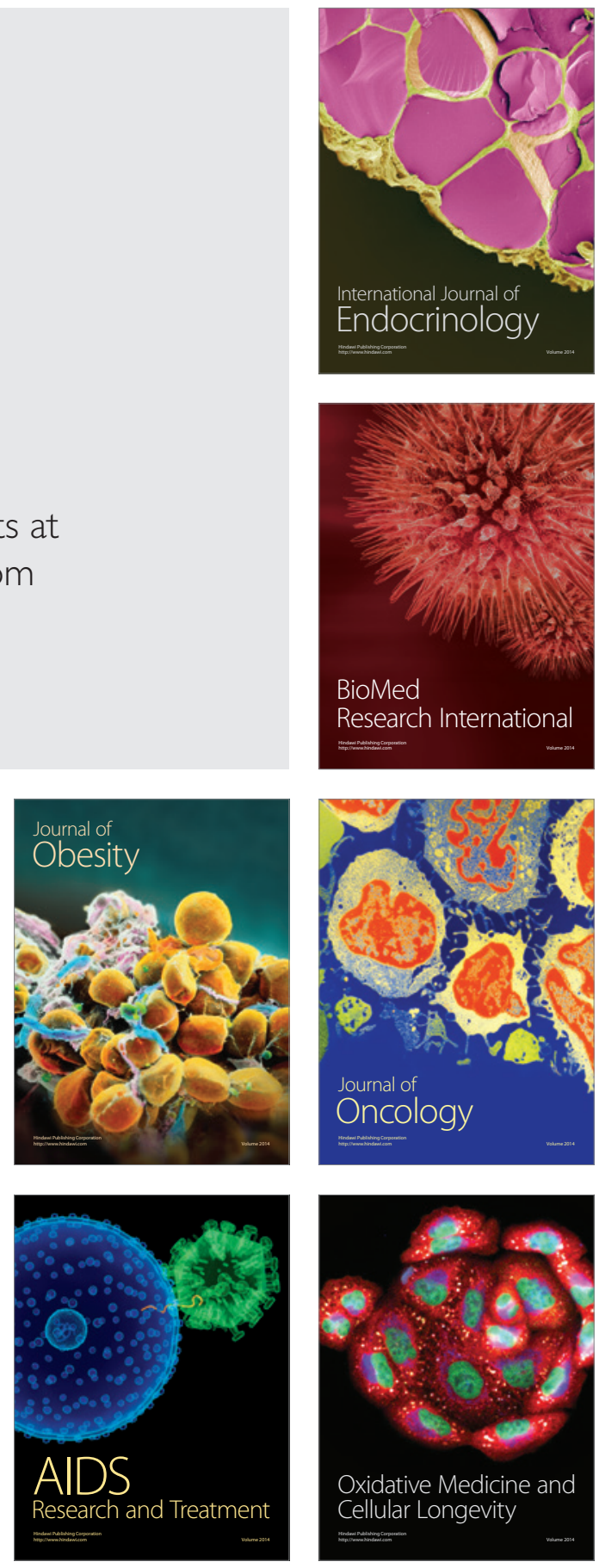